\title{
Anorectal Malformations at a University Teaching Hospital in Southern Nigeria
}

\author{
Nwokoro CC, ${ }^{1}$ Fatungase OM, ${ }^{2}$ Emmanuel EA, ${ }^{2}$ Salami BA, ${ }^{1}$ Ayoade BA, Ogundele IO, Amosu LO. ${ }^{1}$ \\ ${ }^{\prime}$ Department of Surgery and ${ }^{2}$ Department of Anaesthesia and Intensive Care, Olabisi Onabanjo University/Olabisi Onabanjo \\ University Teaching Hospital, Sagamu, Ogun State, Nigeria. \\ *Corresponding Author: Nwokoro C. Olabisi Onabanjo University/Olabisi Onabanjo University Teaching Hospital, Sagamu, Ogun State. \\ Email: collinsnwokoro@gmail.com
}

\begin{abstract}
Ano-rectal malformations comprise a wide spectrum of diseases, which can affect males and females, and involve the distal anus and rectum as well as the urinary and genital tracts. The incidence, type or variety of malformations varies from one region or geographical location to another. Ano-rectal anomalies tend to be associated with other congenital anomalies. The aim of this study is to determine the incidence, presentation and types of ano-rectal malformations and their treatment outcome. It is a prospective observational study from March 2012 to February 2019. Information obtained from cases of ano-rectal anomalies who presented to our health institution during the study period was entered into a proforma. Relevant clinical information was obtained and documented in a structured profoma. A total of 60 patients were seen during the study period. Males were 35 (58.3\%) while females were 25 (41.7\%). Age at presentation ranged from 2 hours -15 years. High anomalies were seen in 34 (56.7\%), low anomalies 18 (30\%) and intermediate 8 (13.3\%). Associated congenital anomalies were seen in $12(20 \%)$ patients. 28 patients had the traditional three-stage procedure of initial colostomy, PSARP and colostomy closure while 13 patients had double staged procedure of initial colostomy and definitive surgery with colostomy closure at the same time. Fifteen (15) patients had single staged procedure (PSARP) without an initial colostomy. Three (3) patients are awaiting colostomy closure after definitive surgery. Post-Operative complications were seen in 16 patients. A mortality of $10(16.7 \%)$ was observed.
\end{abstract}

Keywords:Ano-rectal, Congenital, Malformations, Nigeria, Tertiary, Institution

\section{INTRODUCTION}

A norectal malformation is a common congenital defect. It comprises a wide spectrum of anomalies of the ano-rectal system, urogenital system, sacral spine and perineal musculature. The extent of anomalies in these four components decides the type of ano-rectal malformations. ${ }^{1}$ The etiology of these malformations remains unclear and is likely to be multifactorial. There are however, reasons to believe there is a genetic component. It was recognized as early as 1950 s that there was an increased risk for a sibling of a patient with ARM to be born with a malformation as much as 1 in 100 , compared with the incidence of about 1 in $3500-5000$ in the general population. ${ }^{2}$

Several efforts have been made to appropriately classify or group each anomaly to aid understanding of the pathology and anatomical derangement that is associated 
with each class or group. The various classifications in use include Ladd and Gross (1934), International (1970), Wing spread (1984), Pena (1995) and Krickenbeck (2005)., Based on the above classification methods, three main classes have been identified; they are the high type, intermediate and the low type. The incidence of each variety or class varies from one geo-political area or region to the other. The high anorectal type have been reported to be more common than the low type by several authors. However, some authors have also discovered the low type as the commonest variety in their own regions. $5,6,7,8$

Ano-rectal malformations have been observed to be more common in males than females but some authors have noticed female preponderance in their centers. ${ }^{9,10,11}$

They are responsible for about $67 \%$ of neonatal emergency surgical procedures in developing countries. Therefore, anorectal malformations constitute a significant clinical and surgical load in developing countries not only as emergency procedures but also in terms of definitive corrective surgery and long-term follow-up. ${ }^{12}$

The reason for this research work is to determine the incidence of ano-rectal anomalies in our environment, to characterise the mode of presentation, to identify and classify the common varieties seen in our environment and to determine the outcome of treatment.

\section{MATERIALS AND METHODS}

\section{Study Design}

A prospective observational study of 7 years duration starting from March 2012 to February 2019. Ethical approval was obtained before the commencement of the study. The parents of these children were also duely informed of the research work and the value it would add to the care of their children. The condition of their children and the possible outcome of treatments and complications that may result from the condition itself or the surgical intervention were fully explained to them. Further counseling to the parents was on the meaning of colostomy and its importance in the care of some of these children.

\section{Specific Objectives}

1. To document the clinical types/varieties of anomalies seen in our environment.

2. To classify them and determine the frequency of occurrence of each variety.

3. To document the type of treatment given and the outcome.
4. To identify the types and frequency of associated congenital anomalies.

\section{Inclusion Criteria}

Cases of congenital ano-rectal malformation managed at our centre during the study period.

\section{Exclusion Criteria}

Acquired ano-rectal conditions andcases of congenital anorectal malformation who declined further treatment.

\section{Outcome Measures}

Complications and mortality.

\section{Data Analysis}

Data analysis was done with Statistical Package for Social Sciences (SPSS) version 20.

\section{Methods}

Information obtained from cases of ano-rectal anomalies who presented to our health institution during the study period were entered into a proforma. Data collected were age of patient at presentation, sex, interval between discovery of anomaly and presentation at the hospital, type of anomaly (high, intermediate or low) and varieties of malformations. Diagnosis of these anomalies were made through clinical findings and the use of lateral invertogram. Also included were other associated congenital malformations, type of treatment (initial colostomy, primary anoplasty, anterior sagittal anorectoplasty, posterior sagittal anorectoplasty, anal dilation, anal translocation/transposition, posterior sagittal anorectovaginourethroplasty (PSARVU), operative findings, duration of hospitalization, complications such as soilage, incontinence, constipation, stenosis, anal prolapse, wound dehiscence and number deaths. Patients who survived were discharged to the paediatric surgical out patient clinic and followed-up for at least 2 years from the time of the first intervention.

\section{RESULTS}

Sixty-nine children presented to our centre with congenital ano-rectal malformations during the study period. However, nine patients were not eligible for the study and were excluded from further participation in the study. A total of 60 patients who satisfied the inclusion requirements were 
Table 1: Sex and age at presentation of patients

\begin{tabular}{ccccccc}
\hline SN & Sex & Frequency & $\%$ & Age at Presentation & Frequency & $\%$ \\
\hline 1. & Male & 35 & 58.3 & $0-1$ Week & 47 & 78.3 \\
2. & Female & 25 & 41.7 & $>1$ Week & 13 & 21.7 \\
\hline
\end{tabular}

Table 2: Types and frequency of associated congenital anomalies

\begin{tabular}{llcc}
\hline S/N & Associated Congenital Anomalies & Frequency & $\%$ \\
\hline 1. & Hydronephirosis & 5 & 41.7 \\
2. & Congenital heart anomaly & 4 & 33.3 \\
3. & Hypospadias & 2 & 16.7 \\
4. & Malrotation with vaginal atresia & 1 & 8.3 \\
5. & Spina bifida cystica & 1 & 8.3 \\
\hline
\end{tabular}

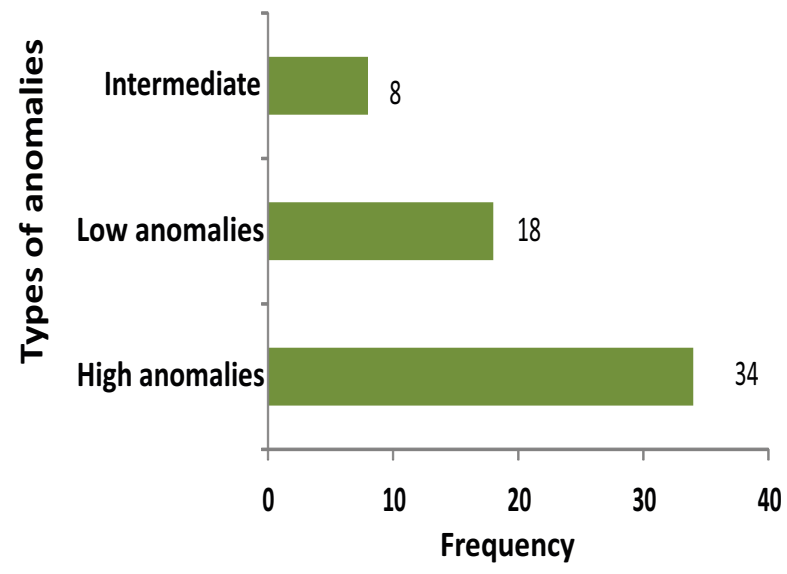

Figure 1: The frequency and types of anorectal anomalies

recruited into the study. Males were 35 (58.3\%) while females were 25 (41.7\%). Those who presented within one week of birth were 47 (78.3\%) while those who presented beyond one week of age were 13 (21.7\%) (Table1). Age at presentation ranged from 2 hours to 15 years. The median age for males was 5 days while the median age for females was 2 months. High anomalies were seen in 34 (56.7\%), low anomalies 18 (30\%) and intermediate 8 (13.3\%) (Figure1). Associated congenital anomalies were seen in $12(20 \%)$ patients. The anomalies were hydronephrosis in five $(41.7 \%)$ patients, congenital heart anomaly in four (33.3\%) patients hypospadias in two patients $(16.7 \%)$, malrotation with vaginal atresia in one patient $(8.3 \%)$, and spina bifida cystica in one (8.3\%) patient, (Table 2). Emergency surgeries were

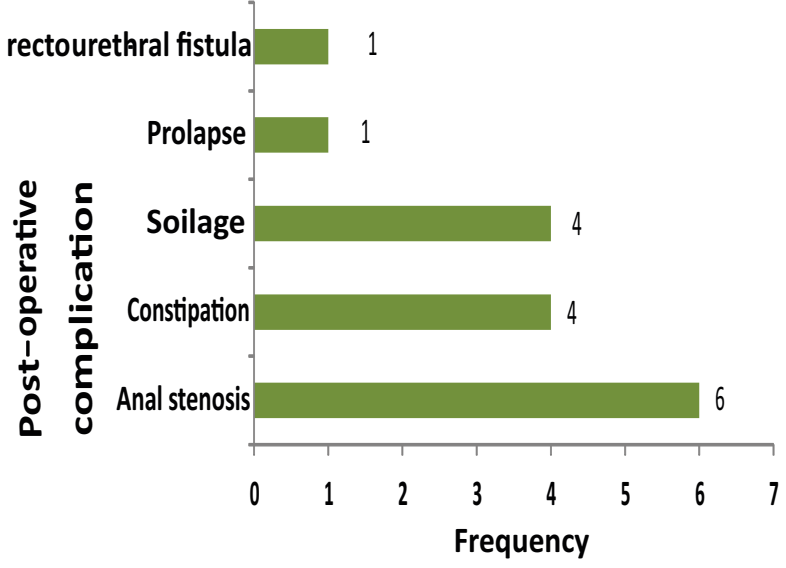

Figure 2: The frequency and post-operative complication

carried out in $42(70 \%)$ patients, elective surgery $18(30 \%)$, primary anoplasty was carried out in $18(30 \%)$ patients, PSARP was done in $42(70 \%)$ patients. 28 patients had the traditional three-stage procedure of initial colostomy, PSARP and colostomy closure while 13 patients had double staged procedure of initial colostomy and definitive surgery with colostomy closure at the same time. Fifteen (15) patients had single staged procedure (PSARP) without an initial colostomy. Three (3) patients are awaiting colostomy closure after definitive surgery. Post-Operative complications were seen in 16 patients. The complications observed in 16 patients were; anal stenosis in six (37.4\%) patients which resolved with anal dilation, four (25\%) patients had constipation which was managed with laxatives, four $(25 \%)$ patients had soilage 
which resolved after a period of toilet training. One (6.3\%) patient had anal prolapse which resolved on sitz bath. One $(6.3 \%)$ patient had recto-urethral fistula and was referred for further evaluation and treatment after failed attempt at repair (Figure 2). A mortality of 10 (16.7\%) was observed. Five were intra-operative following the administration of general anaesthesia (in four male neonates and one female infant), one male infant died post-colostomy of associated congenital heart disease (he was aged 3 days), two male patients (aged 3 months and 6 months) died before colostomy closure from sepsis and two female patients on colostomy (aged one year and two years) died from enteritis while awaiting definitive surgery.

\section{DISCUSSION}

Anorectal malformations comprise of wide spectrum of defects ranging from slight malpositioning of the anus with excellent functional outcomes to complex anomalies of the hindgut and urogenital organs that are difficult to manage. It affects 1 in 4000 to 5000 lives births worldwide with a slight male preponderance. There are variations in overall incidence which may result from environmental, ethnic and genetic influences. ${ }^{13}$ These in turn determine the types and sub-types seen, associated anomalies and different patterns of disease. The anomaly may occur in isolation but is commonly associated with other anomalies. The cause of ARMS has remained largely unknown. However, environmental factors and genetics seem to play a significant role in the occurrence of this condition. ${ }^{13}$

We had a total of 60 patients during the study period but this does not in any way represent the true prevalence of anorectal malformations in our centre because some of the patients in our environment die before reaching our tertiary health facility. In developing and resource limited countries such as ours, awareness is still being created about the availability of several treatment options for patients with anorectal malformations because this condition has been considered as a misfortune without solution in the past. Unidentified and hidden mortalities have also been mentioned by Beudeker and co-workers. ${ }^{11}$

Most of our patients were males and had mostly anorectal malformation with recto-urethral fistula. The female patients presented with imperforate anus and recto-vestibular fistula. This is similar to the results of other workers ${ }^{8,9,10}$. Our patients presented within the first week of life. Delayed presentation (beyond one week of life) was observed in patients who had external fistulae that enabled the passage of feaces and flatus until obstruction necessitated presentation at the hospital. Makanga and co-workers ${ }^{14}$ made similar observations. The high type of anomaly accounted for most of the cases managed in our centre however, other researchers have reported the low-lying anomaly as the majority in their various studies. ${ }^{14,15,16}$

Diagnosis was made at presentation through a combination of clinical features and findings on lateral invertogram. Associated anomalies were mostly urogenital and these occurred in $12(20 \%)$ of our patients. The reported incidence of associated anomalies varies across the world in frequencies as well as type of associated anomalies. The reported range of associated anomalies varies between $20 \%$ and $80 \%$. Most of our diagnosis were made through the use of lateral invertogram, abdomio-perineal ultrasonography, plain abdominal erect radiography and ecocardiography. Other investigative modalities that improved the diagnostic accuracy of anorectal malformations and other associated anomalies are computerized tomography scan of the abdomen and magnetic resonance imaging.

Patients who had low anomalies in our centre were offered primary anoplasty after resuscitation while those with high anomalies had staged management which involved initial colostomy, definitive PSARP and colostomy closure. This is similar to the practice in other centres. Skin excoriation was a major problem to our patients on colostomy because the ideal colostomy bags were not usually available, hence improvised devices were employed to receive fecal effluents from the stoma sites. Some patients were selected for two stage and one stage procedures according to their presentation. These included female patients with anovestibular fistula. Traditionally, high anorectal malformations were managed through a three-stage procedures of initial colostomy, definitive surgery, posterior sagittal anorectoplasty (PSARP) and closure of colostomy. However, this approach is giving way to one stage procedure for both high and low anomalies in some centres with advanced technology in achieving accurate and early diagnosis, instituting prompt intervention and with facilities for intensive care. ${ }^{18}$

The classification of ARMs is mainly based on the position of the rectal pouch relative to the puborectalis sling, the presence or absence of fistulae and the types and location of fistulae. ${ }^{17}$ The information obtained is crucial in determining the most appropriate surgical approach for each case. Imaging 
techniques also play a key role in evaluation and classification of ARMs. In neonates, clinical and radiological examinations in the first 3 days of life help to determine the type of ARM and the need for early colostomy.

The best known classification of ARMs is the Wingspread classification of 1984. It divided ARMs into three types- low, intermediate and high- depending on whether the rectal pouch is located below, at the level or above the puborectal sling.

The surgical approach to repairing these defects changed dramatically in 1980 with the introduction of the posterior sagittal approach which allowed surgeons to view the anatomy of these defects clearly, to repair them under direct vision and to learn about the complex anatomic arrangement of the junction of rectum and gentourinary tract. Better imaging techniques and a better knowledge of the anatomy and physiology of the pelvic structures at birth have refined diagnosis and initial management. ${ }^{4}$

Anorectal malformations have remained a burden in Nigeria, Africa and the whole world as a result of its contribution to neonatal intestinal obstruction with the attendant morbidity and mortality. This condition also presents significant challenges with regards to re-construction, management and long term follow-up. ${ }^{10,12}$

Patients with low anomalies who were critically ill had initial colostomy and subsequently definitive anoplasty and colostomy closure. Complications we observed were constipation, soiling, anal prolapse and recto-urethral fistula. Long term functional outcome in children with ARM, primarily entails bowel function which is of vital importance as fecal incontinence and / or constipation remain major postoperative complications that impede social and psychological development of these patients.

Globally, efforts are being made to standardize the surgical management of children with anorectal malformations. These include the adoption of uniform classification such as was done by Amussat, the father of proctoplasty in 1835 . His work was followed by Ladd and Gross in 1934, producing the First standard classification of this anomaly. ${ }^{10}$ The main concerns for the surgeon in correcting these anomalies are bowel control, urinary control and sexual function. With early diagnosis, management of associated anomalies and efficient meticulous surgical repair, patients have the best chance for a good functional outcome. Fecal and urinary incontinence can occur even with an excellent anatomic repair, due mainly to associated problems such as a poorly developed sacrum, deficient nerve supply and spinal cord anomalies. For these patients an effective bowel management program, including enema and dietary restrictions has been helpful in improving their quality of life. ${ }^{4}$

Further development led to an International classification on the basis of puborectalis muscle, followed by International classification in 1970 and Wingspread classification in 1984. Pena classified ARMS on the basis of presence and position of fistula. Krickenbeck group in 2005 published their findings and incorporated criteria from wing spread and pena's classifications. ${ }^{13}$ Survival and prognosis of anorectal malformations depends upon the severity and number of associated anomalies. Early diagnosis, management of associated anomalies and efficient meticulous repair provide patients the best chance for a good functional outcome. ${ }^{9,14}$

Every child with an anorectal malformation has unique needs. The multidisciplinary care team will develop a treatment plan based on the child's type of anorectal malformation, the presence and type of associated conditions. The multidisciplinary team will develop a treatment plan based on the child's type of anorectal malformation. ${ }^{4}$ The mortality encountered in patients with anorectal malformation was high in the past due to lack of understanding of the nature and anatomy of these malformations, neonatal problems such as hypotemia, hypoglyceamia, poor immunity and sepsis, associated anomalies, delayed presentation and risk of anaesthesia. $^{9,19}$

Our patients mostly presented late and as a result were major anaesthestic and surgical risks. As the resuts showed, we had most of our mortality in the neonatal period. Our overall mortality was $20 \%$. The deaths occurred from anaesthetic complications, sepsis and associated anomalies. However, as a result advancements in diagnostic, therapeutic modalities and availiability of neonatal intensive care units. The morality rate is reduceing and the mortality rate is reported to be around $1.4 \%-30 \%$ in some centres. ${ }^{20}$

\section{CONCLUSION}

Ano-rectal malformations in our environment occurred more in males, the high type was the commonest variety and the mode of surgical treatment was posterior sagittal anorectoplasty for the high/intermediate type and primary anoplasty for the low type. Our center is gradually moving towards the single stage procedure for the intermediate and some selected high anomalies. 


\section{REFERENCES}

1. Upadhyaya V.D. Gangopadhyay A.N., Srivastava P., Zaheer H., Sharma S.P. Int. J of surg 2017;17:1-7.

2. Falcone R.A, Levitt M.A., Bates M.D. Increase heritability of certain types of ano-rectal malformations. J pediatr surg 2007;42:124-128.

3. Nah S.A., Ong C.C., Lakshmi N.K., Yap T.L., Jacobsen A.S., Low Y. Anomalies associated with ano-rectal malformations according to the krickenback anatomic classification. J pediatr surg 2012;47:2273-8.

4. Marc A.M. and Pena A. Ano-rectal malformations. Orpanet j Rare Dis 2007;2:33.

5. Cho S., Moore S.P., Faigman T. One hundred three consecutive patients with ano-rectal malformations and their associated anomalies. arch pediatr Adolesc med 2001;155:587-91.

6. Lukon C.S., Anah E.A., Mshalburala P.A., Jabo B.A., Homa A., Akiniyi O.T., Nmadu P.T. Ano-rectal malformations: changing trend over two decades in Zaria, Nigeria. Afri J paediatr surg 2011;8:19-22.

7. Wakhlu A.K. Management of congenital Ano-rectal malformations. Indian Pediatrics 1995;32:1240-1242.

8. Adekunle O.O. and Johnson A.O.K. congenital ano-rectal anomalies in Western Nigeria. Nigerian journal of paediatrics 1981;8:46.

9. Adeniran J.O., Adejanye A.O. Ano-rectal anomalies in Ilorin, Nigeria a 10-year review. Nigerian journal of surgical research 2003;5:148-151.

10. Moore S.W., Sidler D., Hadley G.P., Ano-rectal malformations in Africa. S Afr J surg 2005;43:175.

11. Beudeker N., Broadis E., Bordstein E., Heil H.A. The hidden mortality of imprforate anus. Afri J paediatr surg 2013;10:302-6.

12. Amen E.A., Dogoand P.M., Mnadu P.T. Emmergency neonatal surgery in a developing country. Pediatr surg inter 2001;17:448-51.

13. Ajay N.G., Vaibhav P. Ano-rectal Malformations. I Indian Asssoc of pediatr surg 2015;20:10-5.

14. Makanga M., Ntirenganya F., Kakande I. Ano-rectral malformations at university teaching hospital of Butare in Rwanda: A reviewe of 46 operative cases. East Cent. Afr. J Surg 2007;12:110-115.

15. Ibrahim D. Ano-rectal malformations Incidence and Significance of associated anomalies. JRMS 2007;14:31-
36.

16. Hese A.A., Appeadu-Mensah W. Ano-rectal Anomalies in Ghana. A Review of 54 cases. Afr J paediatr surg 2006;3:48.

17. Alamo L., Meyrat B.J., Meuwly J.Y., Meuli R.A., Gudinchet F. Ano-rectal malformations: findings the pathway out of the labyrinth. Radiographics 2013;33:491512.

18. Shahjahan M., Karim S. Ferdous KMN, Khan T.A., Rashid M.A. Anomalies Associated with Ano-rectal malformation. Experience in Dhaka Shishu (children) Hospital.

19. Ramadhan J.M., Alicia M., Florentina M., Japhet M.G., Phillipol L.C. Clinical profile and outcome of surgical managment of ano-rectal malformations at a tertiary care hospital in Tanzania. Tanzania journal of health research 2018;20:1-11.

20. Doria F., Amaral D., Ano-rectal anomalies by anterior perinealanorectoplasty. J pediatr surg 1999;34:1315-9. 